\title{
Appendix
}

\section{Commonly Used Approved Abbreviations}

Standard Units of Measurements/Quantities/Allied Terms

\begin{tabular}{|c|c|}
\hline $\begin{array}{l}\text { Abbreviation or } \\
\text { Symbol }\end{array}$ & Term \\
\hline A & ampere \\
\hline$\AA$ & angstrom \\
\hline atm & standard atmosphere \\
\hline $\mathrm{b}$ & barn \\
\hline $\mathrm{bp}$ & base pair \\
\hline $\mathrm{Bq}$ & bacquerel \\
\hline $\mathrm{C}$ & coulomb \\
\hline${ }^{\circ} \mathrm{C}$ & degree Celsius \\
\hline cal & calorie \\
\hline $\mathrm{cd}$ & candela \\
\hline $\mathrm{Ci}$ & curie \\
\hline $\mathrm{cm}^{2}$ & square centimeter \\
\hline cpm & counts per minute \\
\hline cps & counts per second \\
\hline d & day \\
\hline $\mathrm{D}$ & Dalton \\
\hline dpm & disintegrations per minute \\
\hline dps & disintegrations per second \\
\hline $\mathrm{ED}_{50}$ & $50 \%$ effective dose \\
\hline $\mathrm{Eq}$ & equivalent \\
\hline $\mathrm{eV}$ & electron volt \\
\hline $\mathrm{F}$ & Farad \\
\hline g & gram \\
\hline $\mathrm{G}$ & gauss \\
\hline Gy & gray \\
\hline $\mathrm{h}$ & hour \\
\hline $\mathrm{H}$ & henry \\
\hline ht & height \\
\hline $\mathrm{Hz}$ & hertz \\
\hline i.m. & intramuscular \\
\hline i.p. & intraperitoneal \\
\hline IU & international unit \\
\hline i.v. & intravenous \\
\hline $\mathrm{J}$ & joule \\
\hline $\mathrm{K}$ & kelvin \\
\hline$k_{d}$ & dissociation constant \\
\hline$k_{i}$ & inhibition constant \\
\hline$\stackrel{l}{K}_{m}$ & Michaelis constant \\
\hline kat & katal \\
\hline $\mathrm{kb}$ & kilobase \\
\hline kcal & kilocalorie \\
\hline $\mathrm{kD}$ & kilodalton \\
\hline $\mathrm{L}$ & liter \\
\hline $\mathrm{LD}_{50}$ & $50 \%$ lethal dose \\
\hline $\log$ & logarithm, common \\
\hline $\ln$ & logarithm, natural \\
\hline $\mathrm{m}$ & meter \\
\hline $\mathrm{M}$ & molar \\
\hline$M_{\mathrm{r}}$ & molecular weight \\
\hline $\min$ & minute \\
\hline $\mathrm{mL}$ & milliliter \\
\hline$\mu \mathrm{L}$ & microliter \\
\hline mo & month \\
\hline
\end{tabular}

\begin{tabular}{|c|c|}
\hline $\begin{array}{c}\text { Abbreviation or } \\
\text { Symbol }\end{array}$ & Term \\
\hline mol & mole \\
\hline $\mathrm{N}$ & newton \\
\hline $\mathrm{N}$ & normal concentration \\
\hline OD & optical density \\
\hline osmol & osmole \\
\hline $\mathrm{Pa}$ & pascal \\
\hline $\mathrm{PCO}_{2}$ & pressure of $\mathrm{CO}_{2}$ \\
\hline $\mathrm{PO}_{2}$ & pressure of $\mathrm{O}_{2}$ \\
\hline $\mathrm{pH}$ & $\begin{array}{l}\text { negative logarithm of hydrogen } \\
\text { ion activity }\end{array}$ \\
\hline $\mathrm{pI}$ & isoelectric point \\
\hline p.o. & by mouth \\
\hline$R_{f}$ & retardation factor \\
\hline $\mathrm{rad}$ & radiation (ionizing, absorbed dose) \\
\hline $\mathrm{rpm}$ & revolutions per minute \\
\hline RQ & respiratory quotient \\
\hline s & second \\
\hline s.c. & subcutaneous \\
\hline sp act & specific activity \\
\hline sp gr & specific gravity \\
\hline stp & $\begin{array}{l}\text { standard temperature and } \\
\text { pressure }\end{array}$ \\
\hline $\mathrm{Sv}$ & sievert \\
\hline$t_{1 / 2}$ & half-life \\
\hline V & volt \\
\hline$V_{\max }$ & maximum velocity \\
\hline vol & volume \\
\hline vol/vol & volume ratio (volume per volume) \\
\hline $\mathrm{W}$ & watt \\
\hline wk & week \\
\hline wt/vol & weight per volume \\
\hline $\mathrm{wt} / \mathrm{wt}$ & weight ratio (weight per weight) \\
\hline $\mathrm{y}$ & year \\
\hline / & per \\
\hline$\%$ & percent \\
\hline \multicolumn{2}{|c|}{ Combining Prefixes } \\
\hline $\mathrm{T}$ & tera- $\left(10^{12}\right)$ \\
\hline $\mathrm{G}$ & giga- $\left(10^{9}\right)$ \\
\hline M & mega- $\left(10^{6}\right)$ \\
\hline $\mathrm{k}$ & kilo- $\left(10^{3}\right)$ \\
\hline $\mathrm{h}$ & hecto- $\left(10^{2}\right)$ \\
\hline da & deka- $\left(10^{1}\right)$ \\
\hline d & deci- $\left(10^{-1}\right)$ \\
\hline $\mathrm{c}$ & centi- $\left(10^{-2}\right)$ \\
\hline $\mathrm{m}$ & milli- $\left(10^{-3}\right)$ \\
\hline$\mu$ & micro- $\left(10^{-6}\right)$ \\
\hline $\mathrm{n}$ & nano- $\left(10^{-9}\right)$ \\
\hline $\mathrm{p}$ & pico- $\left(10^{-12}\right)$ \\
\hline f & femto- $\left(10^{-15}\right)$ \\
\hline $\mathrm{a}$ & atto- $\left(10^{-18}\right)$ \\
\hline
\end{tabular}




\begin{tabular}{|c|c|c|c|}
\hline $\begin{array}{l}\text { Abbreviation or } \\
\text { Symbol }\end{array}$ & Term & $\begin{array}{l}\text { Abbreviation or } \\
\text { Symbol }\end{array}$ & Term \\
\hline \multicolumn{2}{|c|}{ Statistical Terms } & FFA & free fatty acid \\
\hline ANOVA & analysis of variance & FSH & follicle-stimulating hormone \\
\hline$d f$ & degrees of freedom & GFR & glomerular filtration rate \\
\hline$F$ & variance ratio & $\mathrm{GH}$ & growth hormone \\
\hline$n$ & number of observations & GMP & guanosine $5^{\prime}$-monophosphate \\
\hline NS & not significant & $\mathrm{Hb}$ & hemoglobin \\
\hline $\begin{array}{l}p \\
r\end{array}$ & correlation coefficient & HDL & high density lipoprotein \\
\hline $\mathrm{SD}$ & standard deviation & HEPES & $N$-2-hydroxyethylpiperazine- $N^{\prime}$ - \\
\hline SEM & standard error of the mean & & 2-ethanesufonic acid \\
\hline$t$ test & Student's $t$ test & HIV & human immunodeficiency virus \\
\hline \multicolumn{2}{|r|}{ Other } & \multirow[b]{2}{*}{ HPLC } & leukocyte antigen \\
\hline ACTH & adrenocorticotropin & & $\begin{array}{l}\text { high performance liquid } \\
\text { chromatography }\end{array}$ \\
\hline AIDS & $\begin{array}{l}\text { acquired immunodeficiency } \\
\text { syndrome }\end{array}$ & $\operatorname{Ig}$ & immunoglobulin \\
\hline AMP, ADP, ATP & $\begin{array}{l}\text { adenosine } 5^{\prime} \text {-mono-, di, and } \\
\text { triphosphate }\end{array}$ & $\begin{array}{l}\text { IGF-I (II) } \\
\text { IL }\end{array}$ & $\begin{array}{l}\text { insulin-like growth factor I or II } \\
\text { interleukin }\end{array}$ \\
\hline apo & apolipoprotein & LDL & low density lipoprotein \\
\hline ATPase & adenosine triphosphatase & LH & luteinizing hormone \\
\hline AVP & arginine vasopressin & MAb & monoclonal antibody \\
\hline BSA & bovine serum albumin & mRNA & messenger RNA \\
\hline $\begin{array}{l}\text { cAMP } \\
\text { cDNA }\end{array}$ & $\begin{array}{l}\text { cyclic AMP } \\
\text { complementary DNA }\end{array}$ & NAD, NADH & $\begin{array}{l}\text { nicotinamide adenine dinucleotide, } \\
\text { oxidized and reduced forms }\end{array}$ \\
\hline CNS & central nervous system & NADP, NADPH & nicotinamide adenine \\
\hline $\mathrm{CoA}$ & coenzyme A & & dinucleotide phosphate, \\
\hline DMSO & dimethylsulfoxide & & oxidized and reduced forms \\
\hline DNA & $\begin{array}{l}\text { deoxyribonucleic acid } \\
\text { (deoxyribonucleate) }\end{array}$ & PAGE & $\begin{array}{l}\text { polyacrylamide gel } \\
\text { electrophoresis }\end{array}$ \\
\hline DTT & dithiothreitol & PBS & phosphate-buffered saline \\
\hline EBV & Epstein-Barr virus & PCR & polymerase chain reaction \\
\hline ECG & electrocardiogram & PTH & parathyroid hormone \\
\hline EDTA & ethylenediaminetetraacetate & RIA & radioimmunoassay \\
\hline $\begin{array}{l}\text { EEG } \\
\text { FGTA }\end{array}$ & electroencephalogram & SDS & sodium dodecyl sulfate \\
\hline EGTA & $\begin{array}{l}\text { ethyleneglycol-bis }(\beta \text {-aminoethyl- } \\
\text { ether })-N, N^{\prime} \text {-tetraacetic acid }\end{array}$ & Tris & tris(hydroxymethyl)aminomethane \\
\hline ELISA & enzyme-linked immunosorbent & TSH & thyrotropin \\
\hline & assay & UV & ultraviolet \\
\hline FCS & fetal calf serum & VLDL & very low density lipoprotein \\
\hline
\end{tabular}

Updated January 2000 